\title{
Effects of sugammadex plus rocuronium vs neostigmine plus cisatracurium during renal transplantation on graft function.
}

Maria Vargas ( $\sim$ vargas.maria82@gmail.com )

Universita degli Studi di Napoli Federico II https://orcid.org/0000-0001-7652-970X

\section{Pasquale Buonanno}

Universita degli Studi di Napoli Federico II Dipartimento di Neuroscienze e Scienze Riproduttive ed Odontostomatologiche

\section{Andrea Sica}

Universita degli Studi di Napoli Federico II Dipartimento di Neuroscienze e Scienze Riproduttive ed Odontostomatologiche

\section{Emanuele Sabatella}

Universita degli Studi di Napoli Federico II Dipartimento di Neuroscienze e Scienze Riproduttive ed Odontostomatologiche

\section{Francesco P. D’Alessio}

Universita degli Studi di Napoli Federico II Dipartimento di Neuroscienze e Scienze Riproduttive ed Odontostomatologiche

\section{Simone Alfieri}

Universita degli Studi di Napoli Federico II Dipartimento di Neuroscienze e Scienze Riproduttive ed Odontostomatologiche

\section{Carmine lacovazzo}

Universita degli Studi di Napoli Federico II Dipartimento di Neuroscienze e Scienze Riproduttive ed Odontostomatologiche

\section{Rosa Carrano}

Universita degli Studi di Napoli Federico II

\section{Giuseppe Servillo}

Universita degli Studi di Napoli Federico II Dipartimento di Neuroscienze e Scienze Riproduttive ed Odontostomatologiche

\section{Research article}

Keywords: Renal function, neuromuscular blockade agent, renal transplantation, graft function 
DOI: https://doi.org/10.21203/rs.2.14392/v1

License: (c) (1) This work is licensed under a Creative Commons Attribution 4.0 International License. Read Full License

Version of Record: A version of this preprint was published at Transplantation Proceedings on April 1st, 2021. See the published version at https://doi.org/10.1016/j.transproceed.2020.09.012. 


\section{Abstract}

Background Rocuronium can be used in patients with severe renal failure (creatinine clearance $<30$ $\mathrm{ml} / \mathrm{min}$ ) but the duration of muscle relaxation is longer and results in an increased risk of postoperative residual curarization. Rocuronium can be antagonized by sugammadex but the elimination of the complex they made (rocuronium-sugammadex complex) varies according to the renal function. Two case reports/series reported the use of rocuronium - sugammadex complex during renal transplantation. This retrospective case-control study aimed to investigate the effects of rocuronium - sugammadex, used during the renal transplantation, on transplanted kidney function.

Methods We analyzed 113 medical records of patients undergoing kidney transplantation from January 2015 to December 2018. 47 medical records were excluded because they did not report the administration of rocuronium + sugammadex or cisatracurium + neostigmine during the transplantation. For each medical records the following data were recorded the characteristics of patients and of kidney donor. Blood creatinine, blood urea, blood sodium, blood potassium, blood calcium levels were collected at the day before the surgery, the day of surgery, after 6 hours (hrs), 12 hrs, 24 hrs, 48 hrs and 72 hrs. Diuresis, urinary sodium and urinary potassium levels were collected at the day of surgery, after $6 \mathrm{hrs}, 12$ hrs, 24 hrs, 48 hrs and 72 hrs.

Results We collected data from 66 medical reports. Blood creatinine levels at $6 \mathrm{hrs}, 12 \mathrm{hrs}$ and $24 \mathrm{hrs}$ were significantly lower in roc + sug group than cis + neo group (crea 6 hrs $=0.05$, crea 12 hrs p $=0.038$, crea $24 \mathrm{hrs} \mathrm{p}=0.049$ ). Blood urea levels for $24 \mathrm{hrs}$ after the transplantation were significantly lower in in roc + sug group than cis + neo group (urea 0 hrs $p=0.025$, urea 6 hrs $p=0.011$, urea 12 hrs $p=0.03$, urea 24 hrs $p=0.011$ ). We found no statistically significant differences in blood sodium, blood potassium, blood calcium, diuresis, urinary sodium, urinary potassium levels before and after the transplantation.

Conclusions In this retrospective case-control study, the use of rocuronium and sugammadex during the renal transplant surgery did not affect the recovery of the graft function during the first week after the transplantation.

\section{Introduction}

Rocuronium may be used in patients with severe renal failure (creatinine clearance $<30 \mathrm{ml} / \mathrm{min}$ ) but the duration of muscle relaxation is longer and results in an increased risk of postoperative residual curarization [1,2]. Rocuronium can be antagonized by sugammadex but the elimination of the complex they made (rocuronium-sugammadex complex) varies according to the renal function [3].

In patients with end-stage renal failure, sugammadex $4 \mathrm{mg} / \mathrm{kg}$ is able to clinically reverse deep neuromuscular blockade and it is as rapid as in healthy controls [4]. In a recent study de Souza et al reported that sugammadex $4 \mathrm{mg} / \mathrm{kg}$ resulted in complete reversal of deep rocuronium-induced neuromuscular blockade in patients with normal and severe impaired renal function even if the time from start of sugammadex to recovery of the T4/T1 ratio to 0.9 was slower in the renal group [5]. Different 
studies demonstrated that the pharmacokinetics of sugammadex was significantly altered by chronic renal failure since the kidney is the major excretory organ of this drug [4]. In a phase III study evaluating the PK of rocuronium-sugammadex complex, plasma concentrations of sugammadex dropped slower in the renal failure group compared with the control group [6]. Staals et al reported that "total plasma clearance of sugammadex was 17 times lower and the mean elimination half-life was 16 times higher in the renal failure group" [6]. In case of renal impairment, the rocuronium-sugammadex complex may be retained for a long time since its complete elimination [6]. Rocuronium-sugammadex complex still showed a measurable concentration 7 days after its administration [5]. Considering the prolonged halflife of sugammadex in patients with severe renal impairment, it is not safe to recommend its use in patient suffering of renal failure [5].

Patients with end stage renal failure are candidates for renal transplantation. During renal transplant surgery, anesthesiologists should avoid using nephrotoxic drugs [7]. During renal transplantation muscle relaxation can otherwise be achieved with different drugs eliminated in the presence of renal failure [7]. According to this atracurium and cisatracurium, undergoing Hofmann degradation and ester hydrolysis, may be used. Although rocuronium may be reversed with sugammadex at the end of surgery, excretion of the rocuronium- sugammadex complex is kidney dependent [7]. Actually, the product data sheet does not recommend the use of rocuronium in patient with renal failure until future researches have evaluated its safety and tolerability profile [7]. Two case reports/series without control groups reported the use of rocuronium-sugammadex complex during renal transplantation but they were mainly focused on the evaluation of rocuronium-sugammadex activities $[8,9]$. With these premises in mind, this retrospective study did not have the purpose to evaluate the efficacy of rocuronium-sugammadex, in terms of reversal of neuromuscular blockade, that we know from current literature to be effective even in impaired renal function, this retrospective case-control study aimed to investigate the effects of rocuroniumsugammadex complex used during the renal transplantation on transplanted kidney function.

\section{Methods}

The study was approved by the IRB of Federico II University of Naples, Naples, Italy (protocol number 113/19), and was conducted in compliance with the Declaration of Helsinki (1964 and following amendments), current Good Clinical Practices, and the applicable European and local regulatory requirements.

Patients in our university hospital usually are invited to freely give written informed consent to authorize the use of their clinical data for research purposes. All subjects included in this study provided consent for the use of their data and the local IRB waived the requirement of further written informed consent.

\section{Subjects}

This was a retrospective, case-control study conducted at Federico II University Hospital of male and female patients undergoing general anesthesia for kidney transplantation. 
Inclusion criteria were age $\geq 18$, years, use of sugammadex + rocuronium or neostigmine + cisatracurium during the transplantation, collection of blood, renal and urinary parameters for the first week after the transplantation, no transplant rejection and/or removal of transplanted kidney during the first week. Exclusion criteria were age $<18$ years, transplant rejection or removal of transplanted kidney during the first week and concomitant use of nephrotoxic drugs.

We analyzed 113 medical records of patients undergoing kidney transplantation from January 2015 to December 2018. Forty-seven medical records were excluded because they did not report the administration of rocuronium + sugammadex (roc + sug) or cisatracurium + neostigmine (cis + neo) during the transplantation. All patients received the graft from a cadaveric donor. We finally collected data from 66 medical reports.

\section{Interventions}

Anesthesia was induced with 1.5 to $2.5 \mathrm{mg} / \mathrm{kg}$ propofol and 1 to $2 \mu \mathrm{g} / \mathrm{kg}$ fentanyl. After acceleromyograph calibration, a single dose of $0.6 \mathrm{mg} / \mathrm{kg}$ rocuronium or $0.2 \mathrm{mg} / \mathrm{kg}$ cisatracurium were administered and tracheal intubation was performed. Additional doses of $0.15 \mathrm{mg} / \mathrm{kg}$ rocuronium or 0.03 $\mathrm{mg} / \mathrm{kg}$ cisatracurium were administered during surgery at TOF2 if required. Anesthesia was maintained by sevoflurane and remifentanil and titrated according to hemodynamic and autonomic responses. At the end of surgery, sevoflurane was decreased to an end-tidal concentration of $0.8 \%$ to $1 \%$, and $2 \mathrm{mg} / \mathrm{kg}$ sugammadex or $0.05 \mathrm{mg} / \mathrm{kg}$ neostigmine were administered when the acceleromyograph registered a TOF2 response.

\section{Data collection and outcome}

For each medical records the following data were recorded: age, gender, reason of chronic renal failure, body mass index (BMI), comorbidities, type and dose of neuromuscular blockers and proper reversals, donor age, ischemia time, need of pre and post-operative dialysis, post-operative bleeding. Blood creatinine, blood urea, blood sodium, blood potassium, blood calcium levels were collected at the day before the surgery, the day of surgery, after 6 hours (hrs), 12 hrs, 24 hrs, 48 hrs and 72 hrs. Diuresis, urinary sodium and urinary potassium levels were collected at the day of surgery, after 6 hrs, 12 hrs, 24 hrs, 48 hrs and 72 hrs. Marginal, single or double kidney transplantations, karpinsky score and histologic evaluation of transplanted kidney were collected.

The primary outcome of this study was to evaluate the impact of using rocuronium + sugammadex vs cisatracurium + neostigmine during kidney transplantation on the recovery of kidney function evaluated in terms of serum creatinine and serum urea variations. The secondary outcome was to evaluate if the use rocuronium + sugammadex or cisatracurium + neostigmine were risk factors for kidney failure after kidney transplantation. Acute kidney failure after kidney transplantation was evaluated according KDIGO criteria as increase of creatinine levels of $0.3 \mathrm{mg} / \mathrm{dl}$ after 48 hours. 


\section{Statistical Analysis}

Data are presented as means \pm SDs or median and range interquartile. Data were analyzed with 1-way analysis of variance, and distribution of residuals was investigated with the Shapiro-Wilk test.

Comparison between groups were performed with ANOVA for repeated measurement. Any comparison was weighted according the sample size. The Cohen's d effect size was further calculated on the comparison that reached a statistical significance. The effect size was classified as small $(<0.2)$, medium (0.2-0.8) and large (>0.8). A univariate logistic regression was performed to identify clinically meaningful variables potentially associated with the risk of post-transplantation renal failure. Thereafter, multiple logistic regressions were carried out using backward stepwise variable elimination, including the variables with a $p<0.05$ at the univariate analysis. Statistical significance was set at $p=0.05$. Statistical analysis was performed using SPSS (version 20.0, IBM®, USA).

\section{Results}

Thirty-seven medical records were excluded because they did not report the administration of roc + sug or cis + neo during the transplantation. We finally collected data from 66 medical reports. 30 patients were treated with roc + sug and 36 patients with cis + neo. Table 1 showed the baseline characteristics of enrolled patients. There were no differences in age, gender, BMI, ischemic time, donor's age, creatinine levels and uremic levels at the day before the transplantation, the need of preoperative dialysis and Karpinsky score.

Figure 1 showed the blood creatinine levels before and after the kidney transplantation. Blood creatinine levels at 6 hrs, 12 hrs and 24 hrs were significantly lower in roc + sug group than cis + neo group (crea 6 hrs $=0.05$, crea 12 hrs $p=0.038$, crea $24 \mathrm{hrs} p=0.049$ ). Figure 2 showed the blood urea levels before and after the transplantation. Blood urea levels for $24 \mathrm{hrs}$ after the transplantation were significantly lower in in roc + sug group than cis + neo group (urea 0 hrs $p=0.025$, urea 6 hrs $p=0.011$, urea 12 hrs $p=0.03$, urea $24 \mathrm{hrs} p=0.011$ ). Medium effect sizes were found for the comparisons between groups of the creatinine levels at $6 \mathrm{hrs}, 12 \mathrm{hrs}$ and $24 \mathrm{hrs}$ (Cohen's d crea $6 \mathrm{hrs}=0.51$, Cohen's d crea $12 \mathrm{hrs}=0.53$, Cohen's d crea 24 hrs $=0.50$ ) and of urea levels from 0 to 24 hrs (Cohen's d urea 0 hrs $=0.58$, Cohen's $d$ urea $6 \mathrm{hrs}=0.67$, Cohen's d urea $12 \mathrm{hrs}=0.54$, Cohen's $d$ urea $24 \mathrm{hrs}=0.68$ ). We found no statistically significant differences in blood sodium, blood potassium, blood calcium, diuresis, urinary sodium, urinary potassium levels before and after the transplantation (figure 3 and figure 4).

According to KDIGO criteria an increase of creatinine levels of $0.3 \mathrm{mg} / \mathrm{dl}$ after 48 hours was found in 27 patients of cis + neo group and in 16 patients of roc + sug group. Table 2 showed the univariate and multivariate regression. The use of roc + sug or cis + neo were not independent risk factors for acute kidney failure after the transplantation. In the multivariate analysis the risk of acute kidney failure after the transplantation was independently associated with blood urea level at $48 \mathrm{hrs}$, while the blood creatinine level at $24 \mathrm{hrs}$ was a protective factor. 


\section{Discussion}

To the best of our knowledge this is the first study evaluating the influence of rocuronium and sugammadex, compared with cisatracurium and neostigmine, on graft function after renal transplantation. We retrospectively analyzed medical records of patients undergoing kidney transplantation whose neuromuscular block was reached by cisatracurium or rocuronium and the reversal obtained by neostigmine and sugammadex, respectively. We found that 1 ) during the first 24 hours after the transplantation, blood creatinine and urea levels were significantly lower in rocuronium sugammadex group, 2) no difference in serum and urinary electrolyte levels between the considered groups and 3) the use of both neuromuscular agents and their reversals were not independent risk factors for acute kidney failure after the transplantation.

Creatinine is commonly used as measure of kidney function [10]. The diagnosis of renal failure is usually suspected when serum creatinine in greater than the upper limit of the normal range [10]. In the early post-transplant period the graft function depended also by the donor characteristics [10]. In this study variables that may affect the graft function as the donor's age, the ischemic time and the Karpinski score were not different between the groups and, interestingly, the graft function was well restored during the first 72 hours after the transplantation since the blood creatinine levels decreased as expected [10]. However, this happens much more in sugammadex group even if it is rapidly cleared unchanged via glomerular filtration without tubular secretion, absorption or metabolism [11].

During the renal transplantation surgery, the unclamping of the arterial vessels resulted in reperfusion of the transplanted kidney with an immediate urine production in more than $90 \%$ of patients. This is a proof of the restoration of glomerular function. Theoretically once the glomerular filtration was resumed in the early postoperative period, sugammadex may be easily excreted by the transplanted kidney [12]. In our patients the serum creatinine levels and diuresis were fully restored suddenly after the first 24 hours following the transplantation even in patients treated with rocuronium and sugammadex. This may be due to the fact that rocuronium - sugammadex complex may be removed according their physiologic pharmacokinetics after the restoration glomerular filtration. According to this, there was no increase in serum creatinine levels in rocuronium-sugammadex group. Indeed, the serum creatinine levels were lower in rocuronium group compared with cisatracurium group even if the cisatracurium undergoes Hofmann degradation and ester hydrolysis.

In this study blood urea levels were significantly lower in rocuronium - sugammadex group supporting the good recovery of the graft function. However, we found a slightly increase of blood urea levels during the first 3 days after transplantation. This finding may have many explanations. The transplanted patients received corticosteroids to prevent graft rejection that had a catabolic action which may contribute to serum urea rising $[13,14]$. Furthermore, to counteract acute tubular necrosis (ATN), kidney transplanted patients were treated with diuretic drugs (furosemide) that may increase of urea levels $[15,16,17]$.

Serum and urinary electrolytes didn't show any significant difference in the two groups. The modification of their concentrations was in accordance with the restored kidney function. Potassium excretion was 
demonstrated by its increase in urinary levels and the concurrent decrease of its blood concentrations. Ghoneim et al. reported similar sodium and potassium serum concentrations in pediatric patients undergoing general anesthesia and treated with sugammadex or neostigmine [18].

In this study we found that the use of both neuromuscular agents and their reversals were not independent risk factors for acute kidney failure after the transplantation while proper renal biomarker, as blood creatinine and urea levels, may have a role $[19,20]$. Isik et al. reported an increase of the renal biomarker cystatin $\mathrm{C}$ in patients undergoing general anesthesia treated with neostigmine but not in patients receiving sugammadex for neuromuscular block reversal. The authors concluded that sugammadex showed a higher tolerability than neostigmine. The authors also affirmed that both drugs do not cause renal failure but only a subclinical kidney damage in fact, the effect on kidney function was put in evidence only by cystatin $\mathrm{C}$ but no clinical signs of renal failure or changes in creatinine, blood urea nitrogen, sodium, potassium, and calcium were recorded since in both groups they found a significant reduction in postoperative serum calcium concentration as we did in our study [21].

Detrimental effects of sugammadex on renal function were reported in literature. Histological changes were found and alteration of more sensitive kidney function markers (i.e. N-acetyl-beta-glucosaminidase, microalbuminuria, and beta 2-microglobulin) were observed but in all cases there is no evidence of a clinically evident damage $[22,23,24]$. Other studies didn't confirm the alterations of both classical and new renal damage biomarkers associated to the use of sugammadex and neostigmine [25].

This study has many limitations. We didn't measure hemodynamic changes during surgery and in postoperative period. The graft perfusion can be an important factor affecting transplanted kidney function. However, no significant differences in hemodynamic parameters were reported in previous studies between neostigmine and sugammadex $[26,27,28]$.

Furthermore, since our analysis was retrospectively conducted, we investigated only routine biomarker of renal function; probably more detailed information about graft damage may be drawn by more sensitive biomarkers e.g. NAG, urinary albumin, and beta2-microglobulin.

\section{Conclusion}

In this retrospective case-control study, the use of rocuronium and sugammadex during the renal transplant surgery did not affect the recovery of the graft function in terms of serum creatinine and blood urea increase during the first week after the transplantation. However, prospective and randomized studies are needed to further confirm this fending.

\section{List Of Abbreviations}

rocuronium + sugammadex $=$ roc + sug

cisatracurium + neostigmine $=$ cis + neo 
hours $=$ hrs

body mass index $=\mathrm{BMI}$

\section{Declarations}

\section{Ethics approval and consent to participate}

The study was approved by the IRB of Federico II University of Naples, Naples, Italy (protocol number 113/19). All subjects included in this study provided consent for the use of their data and the local IRB waived the requirement of further written informed consent.

\section{Consent for publication: NA}

\section{Availability of data and materials}

The datasets used and/or analysed during the current study are available from the corresponding author on reasonable request

\section{Competing interest}

The authors have no financial competing interest fro this study

\section{Funding}

The authors received no funding for this study

\section{Authors contributions:}

$\mathrm{MV}, \mathrm{PB}, \mathrm{AS}, \mathrm{Cl}, \mathrm{RC}$ and GS helped to write the protocol of the study, to collect the data, to analyze the data, to write the manuscript and to approve the final version.

ES, FDP and SA helped to collect the data, to analyze the data, to write the manuscript and to approve the final version

\section{References}

1. Robertson EN, Driessen JJ, Booij LH: Pharmacokinetics and pharmacodynamics of rocuronium in patients with and without renal failure. Eur J Anaesthesiol 2005; 22:4-10. 
2. Murphy GS, Szokol JW, Marymont JH, et al.: Residual neuromuscular blockade and critical respiratory events in the postanesthesia care unit. Anesth Analg 2008; 107:130-137.

3. Update on the intraoperative management of adult cadaveric renal transplantation; BJA Education 2016;16:53-57

4. de Souza CM, Tardelli MA, Tedesco H, et al: Efficacy and safety of sugammadex in the reversal of deep neuromuscular blockade induced by rocuronium in patients with end-stage renal disease: $A$ comparative prospective clinical trial. Eur J Anaesthesiol. 2015; 10:681-6.

5. Panhuizen IF, Gold JA, Buerkle C et al. Efficacy, safety and pharmacokinetics of sugammadex $4 \mathrm{mg}$ kg-1 for reversal of deep neuromuscular blockade in patients with severe renal impairment. Br J Anaesth 2015; 114:777-84

6. Staals LM, Snoeck MMJ, Driessen JJ et al. Reduced clearance of rocuronium and sugammadex in patients with severe to end-stage renal failure: a pharmacokinetic study. Br J Anaesth 2010; 104:319

7. Schmid S1, Jungwirth B: Anaesthesia for renal transplant surgery: an update. Eur J Anaesthesiol 2012; 29:552-558.

8. Carlos RV, Torres ML, de Boer HD: The use of rocuronium and sugammadex in paediatric renal transplantation: two case reports. Eur J Anaesthesiol 2016; 33:383-385.

9. Ono A, Fujita Y, Kajiura T, et al.: Efficacy and safety of sugammadex in patients undergoing renal transplantation. JA Clinical Reports 2018; 4:56.

10. Govani MV1, Kwon O, Batiuk TD, Milgrom ML, Filo RS: Creatinine reduction ratio and 24-hour creatinine excretion on post transplant day two: simple and objective tools to define graft function. $J$ Am Soc Nephrol. 2002; 6:1645-9.

11. Cammu G, Van Vlem B, van den Heuvel M, et al.: Dialysability of sugammadex and its complex with rocuronium in intensive care patients with severe renal impairment. Br J Anaesth 2012; 109:382390.

12. Peeters $P$, Passier $P$, Smeets J, et al.: Sugammadex is cleared rapidly and primarily unchanged via renal excretion. Biopharm Drug Dispos 2011; 32:159-167.

13. Wolthers T, Hamberg O, Grøfte T, Vilstrup H.: Effects of budesonide and prednisolone on hepatic kinetics for urea synthesis. J Hepatol. 2000; 4:549-54.

14. Wolthers T, Grøfte T, Jørgensen JO, Vilstrup H.: Growth hormone prevents prednisolone-induced increase in functional hepatic nitrogen clearance in normal man. J Hepatol. 1997; 5:789-95.

15. McCabe R, Stevens LE, Subramamian A, Lattes C, Hashim GM.: Reduction of acute tubular necrosis (ATN) by furosemide and steroids in cadaveric kidney recovery. Am J Surg. 1975; 3:246-8.

16. Nierenberg DW.: Furosemide and ethacrynic acid in acute tubular necrosis. West J Med. 1980; 2:163-70.

17. Peterson OW, Gabbai FB, Myers RR, Mizisin AP, Blantz RC.: A single nephron model of acute tubular injury: role of tubuloglomerular feedback. Kidney Int. 1989; 6:1037-44. 
18. Ghoneim AA, El Beltagy MA: Comparative study between sugammadex and neostigmine in neurosurgical anesthesia in pediatric patients. Saudi J Anaesth, 2015; 9: 247-52.

19. Lemmens HJ, El-Orbany MI, Berry $\mathrm{J}$ et al.: Reversal of profound vecuronium-induced neuromuscular block under sevoflurane anesthesia: Sugammadex versus neostigmine. BMC Anesthesiol, 2010; 10: 5.

20. Rahe-Meyer N, Fennema H, Schulman S et al.: Effect of reversal of neuromuscular blockade with sugammadex versus usual care on bleeding risk in a randomized study of surgical patients. Anesthesiology, 2014; 121: 969-77.

21. Isik Y, Palabiyik O, Cegin BM, Goktas U, Kati I: Effects of Sugammadex and Neostigmine on Renal Biomarkers. Med Sci Monit. 2016 Mar 10; 22:803-9.

22. Bostan H, Kalkan $Y$, Tomak $Y$ et al: Reversal of rocuronium-induced neuromuscular block with sugammadex and resulting histopathological effects in rat kidneys. Renal Failure, 2011; 33: 101924.

23. Sparr HJ, Vermeyen KM, Beaufort AM et al.: Early reversal of profound rocuronium-induced neuromuscular blockade by sugammadex in a randomized multicenter study efficacy, safety and pharmacokinetics. Anesthesiology, 2007; 106: 935-43.

24. Sorgenfrei IF, Norrild K, Larsen PB et al.: Reversal of rocuronium-induced neuromuscular block by the selective relaxant binding agent sugammadex: A dose-finding and safety study. Anesthesiology, 2006; 104: 667-74.

25. Khuenl-Brady KS, Wattwil M, Vanacker BF et al.: Sugammadex provides faster reversal of vecuronium-induced neuromuscular blockade compared with neostigmine: A multicenter, randomized, controlled trial. Anesth Analg, 2010; 110: 64-73.

26. Sparr HJ, Vermeyen KM, Beaufort AM et al.: Early reversal of profound rocuronium-induced neuromuscular blockade by sugammadex in a randomized multicenter study efficacy, safety and pharmacokinetics. Anesthesiology, 2007; 106: 935-43.

27. Tas $\mathrm{N}$, Korkmaz H, Yagan O, Korkmaz M: Effect of Sugammadex on postoperative bleeding and coagulation parameters after septoplasty: A randomised prospective study. Med Sci Monit, 2015; 21: 2382-86.

28. Flockton EA, Mastronardi P, Hunter JM et al.: Reversal of rocuronium-induced neuromuscular block with sugammadex is faster than reversal of cisatracurium-induced block with neostigmine. $\mathrm{Br} J$ Anaesth, 2008; 100: 622-30.

\section{Tables}




\begin{tabular}{|l|c|c|}
\hline & Roc + Sug (30 pts) & Cis + Neo (36 pts) \\
\hline Age (yr) & $52(39-59)$ & $50(41-61)$ \\
\hline Gender (M/F) & $28 / 8$ & $21 / 9$ \\
\hline BMI & $26(24-30)$ & $24(23-30)$ \\
\hline Donor's age (yr) & $49(40-56)$ & $48(41-56)$ \\
\hline Ischemic time (hr) & $10(8-14)$ & $9(7-14)$ \\
\hline Karpinski score (pts) & 4 & 3 \\
\hline Marginal transplantation & 0 & 1 \\
\hline Single transplantation & 1 & 1 \\
\hline Double transplanation & 0 & 0 \\
\hline Creatinine (mg/dl) & $7.95(5.65-11.25)$ & $7.1(5,4-9.10)$ \\
\hline Urea (mg/dl) & $26.5(14-72)$ & $60(42-79)$ \\
\hline Need of dialysis (pts) & 2 & 1 \\
\hline
\end{tabular}

Table 1: characteristics of included patients collected at the day of the kidney transplantation. Pts: patients, Roc + Sug: rocuronium + sugammadex, Cis + Neo: cisatracurium + neostigmine, yr: years, M/F: male/ female, hr: hours, pts: points. Data were reported as frequencies, median and range interquartile.

\begin{tabular}{|l|l|l|l|l|}
\hline \multicolumn{5}{|c|}{ Regression coefficients } \\
\hline & Univariate Analysis & $\mathrm{p}$ & Multivariate Analysis & $\mathrm{p}$ \\
\hline & $0.062(0.021-0.104)$ & 0.004 & $\begin{array}{l}-0.117(-0.223- \\
-0.011)\end{array}$ & 0.031 \\
\hline Creatinine 24 hr & & & & \\
\hline Creatinine 48 hr & $0.082(0.050-0.115)$ & 0.000 & & \\
\hline Creatinine 72 hr & $0.071(0.039-0.103)$ & 0.000 & & \\
\hline Urea 48 hr & $0.003(0.001-0.005)$ & 0.005 & $0.005(0.001-0.009)$ & 0.016 \\
\hline Urea 72 hr & $0.002(0.000-0.004)$ & 0.04 & & \\
\hline $\begin{array}{l}\text { Dialysis post } \\
\text { transplantation }\end{array}$ & $0.422(0.188-0.655)$ & 0.001 & & \\
\hline Na 6 hr & $-0.050(-0.086-$ & 0.009 & & \\
\hline Na 12 hr & $-0.013)$ & & & \\
\hline Na 24 hr & $-0.045(-0.08--0.011)$ & 0.011 & & \\
\hline K 0 hr & $-0.041(-0.074-$ & 0.014 & & \\
\hline K 6 hr & $-0.008)$ & & & \\
\hline K 24 hr & $0.126(0.005-0.247)$ & 0.042 & & \\
\hline Diuresis day 1 & $0.221(0.040-0.401)$ & 0.017 & & \\
\hline Diuresis day 2 & $0.025(0.000-0.050)$ & 0.048 & & \\
\hline Diuresis day 3 & $0.000(0.000-0.000)$ & 0.009 & & \\
\hline Diuresis day 4 & $0.000(0.000-0.000)$ & 0.001 & & \\
\hline Diuresis day 5 & $0.000(0.000-0.000)$ & 0.001 & & \\
\hline Diuresis day 6 & $0.000(0.000-0.000)$ & 0.008 & & \\
\hline Diuresis day 7 & $0.000(0.000-0.000)$ & 0.002 & & \\
\hline
\end{tabular}

Table 2: univariate and multivariate analyses for the risk of post-transplantation renal failure. 
Figures

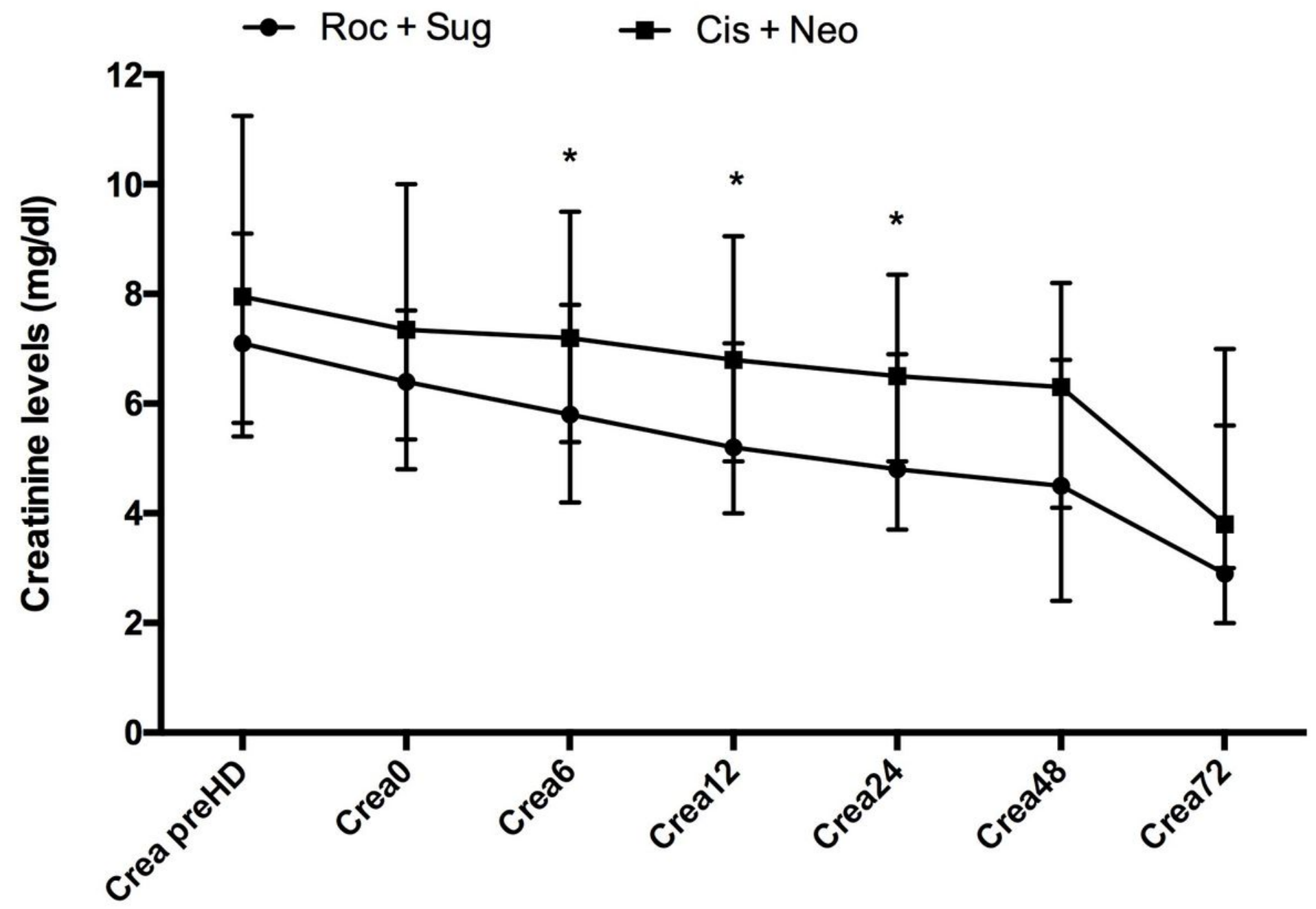

Figure 1

blood creatinine levels before and after the kidney transplantation. Crea: creatinine, roc + sug: rocuronium + sugammadex, cis + neo: cisatracurium + neostigmine. ${ }^{*} p<0.05$. 


\section{$\rightarrow \mathrm{Cis}+\mathrm{NeO} \rightarrow \mathrm{Roc}+\mathrm{Sug}$}

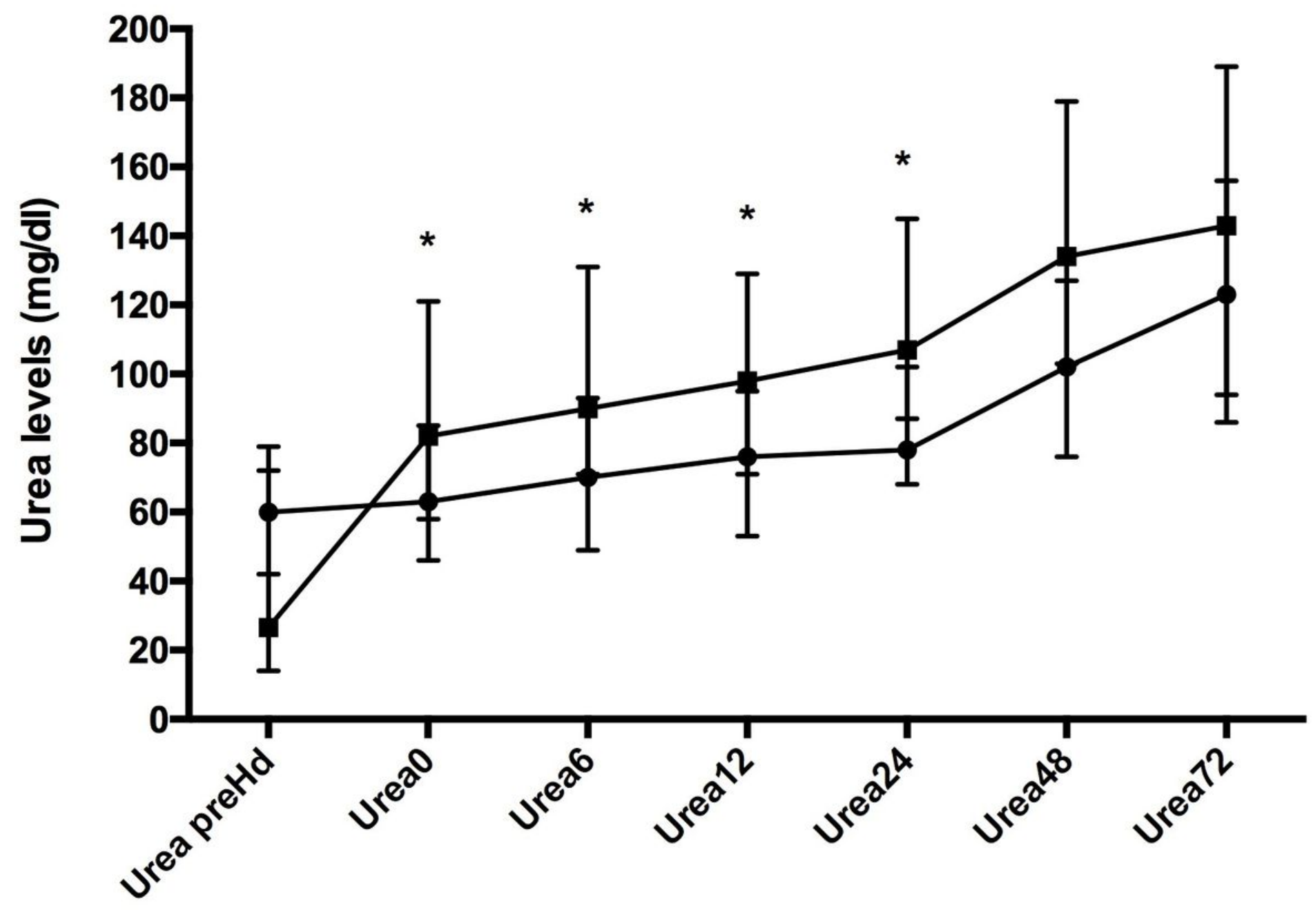

Figure 2

blood urea levels before and after the kidney transplantation. Crea: creatinine, roc + sug: rocuronium + sugammadex, cis + neo: cisatracurium + neostigmine. ${ }^{*} p<0.05$. 


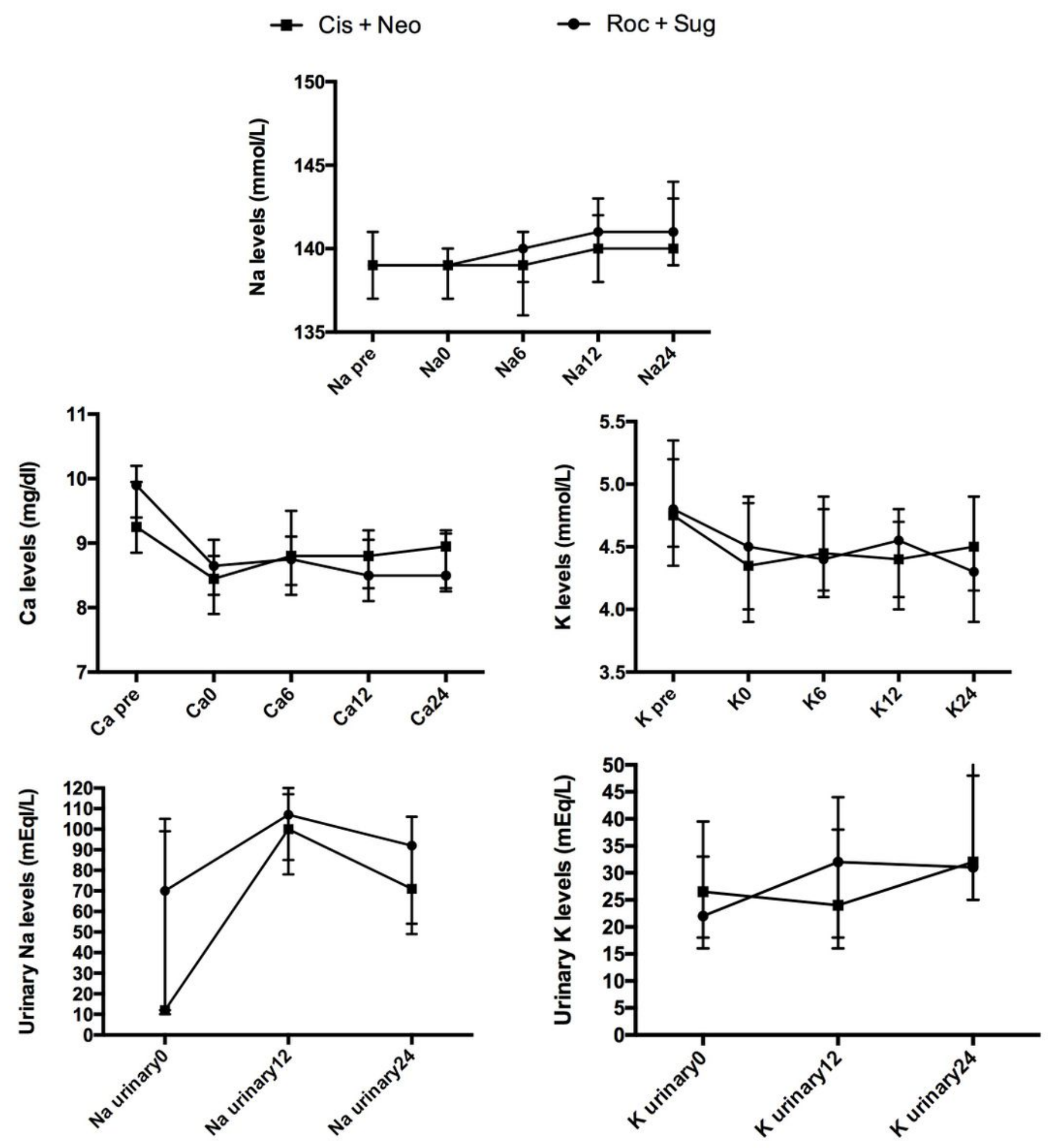

Figure 3

blood sodium, blood potassium, blood calcium levels and urinary sodium and urinary potassium levels before and after the kidney transplantation. Crea: creatinine, roc + sug: rocuronium + sugammadex, cis + neo: cisatracurium + neostigmine. 


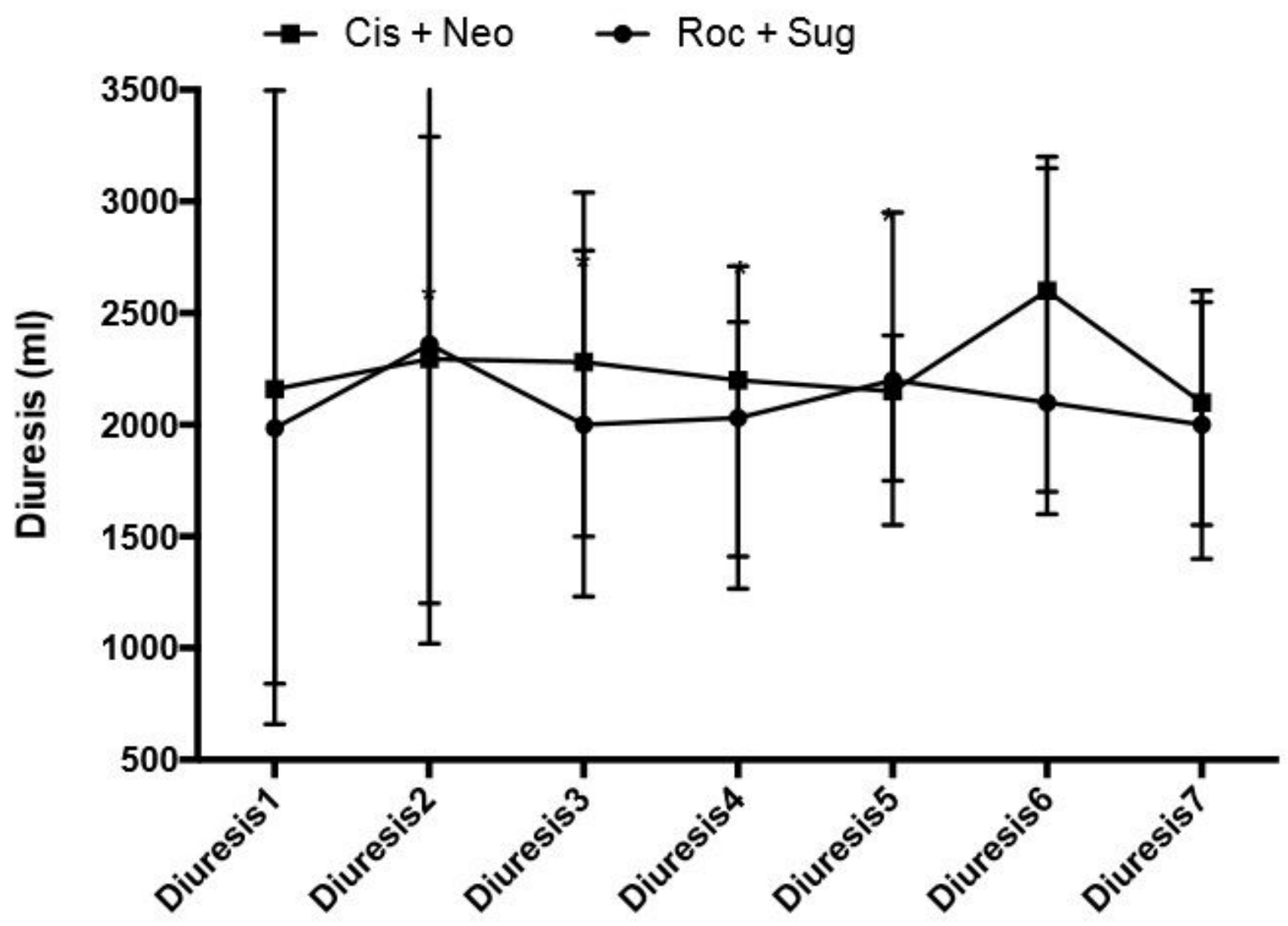

Figure 4

Diuresis levels before and after the kidney transplantation. Crea: creatinine, roc + sug: rocuronium + sugammadex, cis + neo: cisatracurium + neostigmine. 